
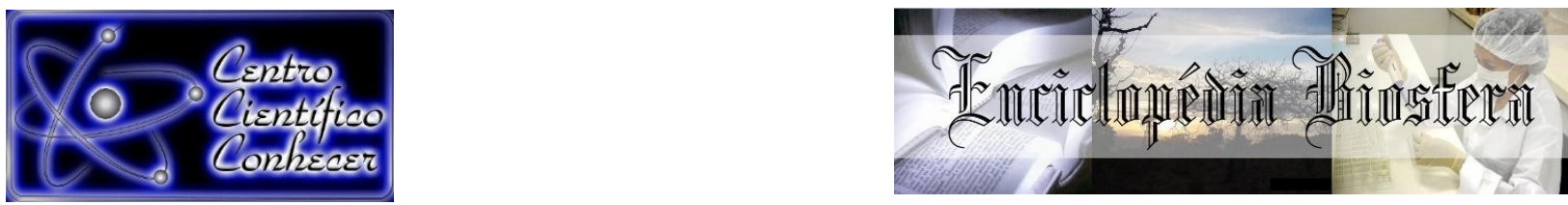

\title{
ASSOCIAÇÃO ENTRE O CONSUMO ALIMENTAR E FATORES DE RISCO CARDIOMETABÓLICOS EM UMA AMOSTRA DA POPULAÇÃO DE SERVIDORES DA UNIVERSIDADE FEDERAL DO TOCANTINS
}

\author{
Josiane de Alcântara Buzachi Garcia, Elisama Costa Lopes², Tainara Pereira \\ Araújo $^{3}$, Renata Junqueira Pereira ${ }^{4}$
}

1 Nutricionista pela Universidade Federal do Tocantins

2 Mestre em Ciências da Saúde pela Universidade Federal do Tocantins

3 Mestranda em Ciências da Saúde pela Universidade Federal do Tocantins

4 Doutora em Ciência dos Alimentos e docente do curso de Nutrição da Universidade

Federal do Tocantins. Autora para correspondência. E-mail: renatajunqueira@uft.edu.br

\section{Recebido em: 15/02/2020 - Aprovado em: 15/03/2020 - Publicado em: 30/03/2020} DOI: 10.18677/EnciBio_2020A4

\begin{abstract}
RESUMO
Introdução: A presença simultânea de fatores como obesidade, hipercolesterolemia, hipertrigliceridemia, hipertensão arterial e resistência insulínica é denominada Síndrome Metabólica e está associada aos hábitos de vida da população, determinados pelas necessidades da "vida moderna". Objetivo: realizar triagem clínica nutricional de 14 servidores da Universidade Federal do Tocantins e confrontar o consumo alimentar com as recomendações nutricionais para adultos saudáveis, associando as inadequações de consumo alimentar à presença de fatores de risco cardiometabólicos. Método: Foi realizada anamnese detalhada, para coleta de dados sociodemográficos, antropométricos e de consumo alimentar. Os dados foram analisados nos softwares DietBox e Microsoft Office Excel. Resultados: $71,4 \%$ dos participantes eram casados, distribuídos equitativamente entre os sexos (50\%), com idades entre 27 e 42 anos. Todos os participantes relataram que nunca fumaram e $50 \%$ relataram consumir bebida alcoólica. Em relação à prática regular de atividades físicas, a maioria era sedentária $(57,2 \%)$. No que se refere ao estado nutricional, $42,86 \%$ apresentaram sobrepeso/obesidade, $50 \%$ estavam eutróficos e 7,14\% apresentaram magreza de grau III. O perímetro da cintura revelou $35,70 \%$ dos indivíduos em risco cardiometabólico muito elevado e $14,28 \%$ apresentaram alteração da glicemia de jejum. A média de consumo energético foi de 1651,92 Kcal, as ingestões de proteína e lipídio estiveram dentro das recomendações. Apresentaram-se superiores às recomendações, as ingestões diárias de retinol, tiamina, niacina, ácido ascórbico, ferro, selênio e fósforo. Conclusão: Identificou-se realidade de saúde desfavorável, quando analisados parâmetros antropométricos e dietéticos dos participantes, afirmados pelos exames bioquímicos, que revelaram um público predisposto a doenças cardiovasculares e crônicas não transmissíveis.
\end{abstract}

PALAVRAS-CHAVE: Consumo de alimentos, deficiências nutricionais, síndrome metabólica. 


\title{
ASSOCIATION OF FOOD CONSUMPTION WITH CARDIOMETABOLIC RISK FACTORS IN AN ADULT POPULATION
}

\begin{abstract}
Introduction: the simultaneous presence of factors such as obesity, hypercholesterolemia, hypertriglyceridemia, high blood pressure and insulin resistance is called Metabolic Syndrome and is linked to changes in the habits of the population, determined by the needs of "modern life". Objective: to perform nutritional clinical screening of fourteen employees of the Universidade Federal do Tocantins and to compare dietary intake with nutritional reference recommendations for healthy adults, associating the inadequacies of dietary intake with the presence of cardiometabolic risk factors. Method: A detailed anamnesis was performed to collect sociodemographic, anthropometric and food consumption data. The data was analyzed in the DietBox and Microsoft Office Excel softwares. Results: $71.4 \%$ of the participants were married, distributed equally between the sexes (50\%), aged between 27 and 42 years. All participants reported they had never smoked and $50 \%$ reported consuming alcoholic beverage. Concerning regular physical activity; the majority is considered sedentary $(57.2 \%)$. Regarding the nutritional status, $42.86 \%$ were overweight / obese, $50 \%$ were eutrophic and $7.14 \%$ presented three grade thinness. The waist circumference revealed $35.70 \%$ of individuals at very high cardiometabolic risk and $14.28 \%$ presented fasting blood glucose alterations. The mean energy consumption of the 24-hour recall was $1651.92 \mathrm{Kcal}$, protein and lipid intakes were within the recommendation. The daily intakes of retinol, thiamine, niacin, ascorbic acid, iron, selenium and phosphorus were higher than recommendations. Conclusion: An unfavorable health situation was identified when the anthropometric and dietary parameters of the servers participating in the study were analyzed, confirmed by biochemical tests, which revealed a population predisposed to cardiovascular diseases and non-communicable chronic diseases.
\end{abstract}

KEYWORDS: Deficiency diseases, food consumption, metabolic syndrome.

\section{INTRODUÇÃO}

A Organização Mundial de Saúde (OMS) determina que os principais fatores de riscos associados às doenças e agravos não transmissíveis (DANT) são pressão arterial elevada, hipercolesterolemia, ingestão insuficiente de frutas e vegetais, inatividade física, excesso de peso ou obesidade e tabagismo (VUORI, 2018). A expressão risco cardiometabólico refere-se à situação em que um ou mais dos fatores de risco aparecem juntos. A presença simultânea de fatores tais como obesidade, hipercolesterolemia, hipertrigliceridemia, pressão arterial elevada e resistência insulínica é conhecida como Síndrome Metabólica, sendo considerada uma pandemia que afeta principalmente mulheres (RAMIRES et al., 2018).

Atualmente, as DANT são responsáveis por $63 \%$ da carga mundial de doenças e a presença dos fatores de risco para DANT mostra-se elevada no Brasil (MALTA et al., 2019). Mudanças têm ocorrido nos hábitos de vida da população, determinadas sobretudo pelas necessidades do estilo de vida atual adotado pela população. Tais necessidades levam a mulher a sair de casa para competir no mercado de trabalho, refletindo em falta de tempo para preparar alimentos, modificando, como consequência, o padrão alimentar, o gasto energético e o estado nutricional de toda a família (LELIS et al., 2012). Segundo a Pesquisa de Orçamentos Familiares (POF 2017-2018) o consumo de gorduras saturadas e trans da população brasileira encontra-se próximo ao limite máximo tolerável, enquanto o consumo de fibras situa-se próximo ao limite 
mínimo recomendado (BRASIL, 2011a). Os teores de açúcar e sódio na alimentação estão, respectivamente, $50 \%$ e $100 \%$ superiores aos limites máximos da ingestão recomendada (VUORI, 2018).

No entanto, hábitos alimentares saudáveis e a prática de atividades físicas exercem um efeito protetor sobre o desenvolvimento de doenças e na promoção da saúde. Intervenções relacionadas à promoção da saúde e à prevenção e controle da obesidade e dos fatores de risco cardiovasculares, tais como o incentivo à prática de atividades físicas, ao abandono do tabagismo, ao equilíbrio da alimentação, ao aumento do consumo de frutas e hortaliças e à adoção de estilos de vida mais saudáveis têm recebido grande importância, por resultarem em redução do peso, dos níveis plasmáticos de lipídeos, de glicose e de pressão arterial, reduzindo o risco cardiometabólico (CHAVES et al., 2015).

Considerando os cenários para intervenções nutricionais, o local de trabalho mostra-se como ambiente estratégico para promover e apoiar ações de promoção, prevenção e controle das DANT, possibilitando ações contínuas e em diferentes níveis - individual, interpessoal, ambiental e organizacional (BRAVIM et al., 2016).

O sobrepeso e a obesidade estão relacionados à redução da capacidade para esforços físicos e à fadiga no desempenho de tarefas, com impactos sobre a motivação do indivíduo (BRAVIM et al., 2016). Atividades promotoras de saúde no ambiente de trabalho melhoram não apenas a qualidade de vida dos trabalhadores, mas também a produtividade.

O objetivo deste estudo foi realizar triagem clínico nutricional e coletar dados do consumo alimentar, antropometria, exames bioquímicos, perfil pressórico e fatores comportamentais de 14 servidores da Universidade Federal do Tocantins, para caracterizar o consumo alimentar em relação às recomendações dietéticas de referência para adultos saudáveis e associá-lo à presença de fatores de risco cardiometabólicos.

\section{MATERIAL E MÉTODOS}

Foi realizado estudo transversal, com servidores, pacientes do complexo ambulatorial do curso de Nutrição da Universidade Federal do Tocantins - UFT, campus de Palmas. Quatorze pacientes foram acompanhados nos atendimentos ambulatoriais de nutrição e completaram anamnese detalhada, para coleta de dados sociodemográficos (sexo, idade, estado civil), econômicos, motivo da consulta, hábitos de vida (consumo de bebida alcoólica, bem como a frequência, tabagismo, prática de atividade física), consumo alimentar (recordatório de 24 horas - R24h), preferências alimentares, hábitos gastrintestinais e avaliação antropométrica.

O consumo alimentar e a ingestão de energia de cada participante foram avaliados por meio da aplicação de um R24h, com a utilização de utensílios de medidas caseiras e álbum fotográfico de porções de alimentos. Os dados da ingestão de nutrientes foram calculados utilizando-se o software DietBox, a partir das tabelas de composição de alimentos que o programa contempla, sendo: Tabela Brasileira de Composição de Alimentos (TACO) (NEPA, 2011), Tabela de Composição Nutricional dos Alimentos Consumidos no Brasil (BRASIL, 2011b), Tabela elaborada pelo United States Departament of Agriculture (USDA, 2012) e Tabela de Composição de alimentos: suporte para decisão nutricional (PHILIPI, 2015).

Quanto aos hábitos alimentares, foram coletados: número de visitas ao supermercado por mês; tempo gasto para realizar a principal refeição (em minutos); padrões de consumo de açúcar e adoçantes; adição de sal à refeição já pronta; tipo de gordura utilizada no preparo das refeições; consumo diário de água; autoavaliação da 
qualidade do sono; preferências e aversões alimentares; consumo de fast foods; presença de doenças do trato gastrintestinal; presença de alergias alimentares e utilização de medicamentos. A prática de atividades físicas foi classificada conforme preconizado pelo Center for Disease Control (CDC) (ARAÚJO; REZENDE, 2019), sendo considerado indivíduo ativo, aquele que pratique qualquer atividade física por pelo menos 150 minutos na semana.

Para obtenção do Índice de Massa Corporal (IMC) foram aferidos o peso corporal e a estatura, conforme descrito por Lopes e Rezende (2019). Para aferição do peso corporal foi utilizada uma balança digital, da marca Welmy, com capacidade máxima de $300 \mathrm{~kg}$, sendo que cada indivíduo portava roupas leves e encontrava-se descalço, posicionado no centro da balança, com os braços dispostos ao longo do corpo. Para aferição da estatura foi utilizado um estadiômetro, acoplado à parede, com o participante ereto, encostado posteriormente à parede, com os pés unidos e os braços dispostos ao longo do corpo. Para classificação do estado nutricional foram usados os pontos de corte para IMC propostos pela Organização Mundial da Saúde (OMS) (LOPES; REZENDE, 2019).

O risco cardiometabólico foi identificado pela medida do perímetro da cintura $(\mathrm{PC})$, aferido com fita métrica flexível e inelástica, com extensão de 1,5 metros, em duplicata, sem comprimir a pele, segundo as técnicas descritas por Lopes e Rezende (2019). O PC foi aferido ao final de uma expiração normal, no ponto médio entre a margem inferior da última costela e a crista ilíaca, os pontos de corte utilizados foram os propostos pela OMS (LOPES; REZENDE, 2019). Foram realizadas determinações sanguíneas de glicemia de jejum, colesterol total (CT) e triacilglicerois (TG), por laboratório privado de análises clínicas da cidade de Palmas, que coletou as amostras de sangue e realizou as determinações bioquímicas.

Para a avaliação da glicemia de jejum adotou-se a referência preconizada pela Diretriz da Sociedade Brasileira de Diabetes (OLIVEIRA et al., 2017). Para a avaliação do lipidograma adotaram-se os valores de referência dispostos na Atualização da Diretriz Brasileira de Dislipidemias e Prevenção da Aterosclerose (FALUDI et al., 2017).

Os dados coletados foram tabulados no Microsoft Excel e analisados no Statistical Package for Social Sciences (SPSS) versão 20.0. Os servidores participantes do estudo assinaram o Termo de Consentimento Livre e Esclarecido. O trabalho foi submetido ao Comitê de Ética em Pesquisa da Universidade Federal do Tocantins e aprovado com o parecer número 1.857.482.

\section{RESULTADOS E DISCUSSÃO}

Dentre os participantes avaliados no estudo, a maioria eram casados $(71,4 \%)$, sendo $50 \%$ do sexo feminino. As idades dos servidores variaram entre 27 e 42 anos. Todos os participantes afirmaram nunca haverem fumado, a ausência de participantes tabagistas neste estudo reflete a redução do tabagismo que vem sendo observada no Brasil, possivelmente impulsionada por políticas restritivas (AUDI et al., 2016).

Metade dos participantes consumia bebida alcoólica, com frequência de consumo variando de uma a três vezes por semana. Dentre os efeitos negativos associados ao consumo de bebida alcoólica estão o aumento da pressão arterial, e aumento do depósito de gordura abdominal e da circunferência da cintura (NEUENSCHWANDER et al., 2019). Quanto à prática de atividade física, 42,8\% dos participantes se classificaram como indivíduos ativos, enquanto $57,2 \%$ não praticavam atividade física regular. 
A prática de atividade física está vinculada à prevenção de doenças crônicas não transmissíveis (DCNT), que acometem grande parte da população como: diabetes tipo 2, hipertensão arterial, obesidade, aterosclerose, osteoporose entre outras (ARAÚJO; REZENDE, 2019). Adicionalmente, a prática regular de atividade física proporciona condicionamento físico e melhor qualidade de vida, além de oferecer benefícios na aparência, no trabalho, na saúde, no dia-a-dia e nos aspectos psicológicos (FERREIRA et al., 2015).

Com referência ao histórico alimentar, todos os participantes faziam compras semanais, priorizando a aquisição de vegetais frescos, refletindo uma preocupação com a inclusão de frutas e verduras às refeições. Relataram também o consumo de produtos in natura em detrimento aos produtos industrializados. As compras organizadas semanalmente refletem um planejamento que, além de gerar impacto positivo na saúde, também contribui para a economia doméstica, limitando a aquisição de alimentos supérfluos, priorizando um cardápio planejado e evitando o desperdício (MENDES et al., 2018).

Sobre o tempo gasto para realizar a principal refeição do dia, todos os participantes tinham o almoço como principal refeição, sendo que $50 \%$ dos participantes comiam muito rápido (de 12 a 15 minutos), 35,71\% levavam entre 15 a 20 minutos para comer e $14,28 \%$ relataram comer com calma e mastigar bem os alimentos, levando mais de 20 minutos para realizar o almoço. É importante considerar que o tempo é reflexo do cotidiano de cada um e que o estilo de vida atual não tem contribuído para que os indivíduos desfrutem de momentos relaxantes e agradáveis na hora das refeições. Ao contrário, o horário de almoço, para a maioria dos trabalhadores, significa enfrentar o trânsito, dedicar-se aos filhos e preparar os alimentos em tempo hábil de retornar para o turno vespertino.

Apesar da importância da mastigação no processo digestivo, estes fatores relacionados à rotina moderna influenciam o tempo dedicado à refeição, negligenciando a mastigação. A boa mastigação tem íntima relação com as sensações inerentes à ingestão de alimentos como fome, apetite e saciedade. Durante a refeição, a sensação de fome vai se atenuando gradualmente e os reflexos para ingestão de alimentos vão sendo inibidos, refletindo em menor ingesta alimentar, quando a refeição é realizada no tempo adequado (MENEGASSI, 2017).

As taxas de sobrepeso e obesidade vêm aumentando nos últimos anos, sendo essa uma tendência que se reflete também nos trabalhadores (BRAVIM et al., 2016). $\mathrm{Na}$ amostra estudada, $42,86 \%$ dos indivíduos apresentaram sobrepeso e/ou obesidade (Figura 1). Apesar do elevado percentual de excesso de peso encontrado, essa taxa ainda está inferior aos dados levantados pela Pesquisa de Orçamentos Familiares 2008-2009, que revelou que o sobrepeso e a obesidade atingiram mais da metade dos adultos em todas as regiões, inclusive na região Norte (58,3\% dos homens, $61,9 \%$ das mulheres) (BRASIL, 2011a). 


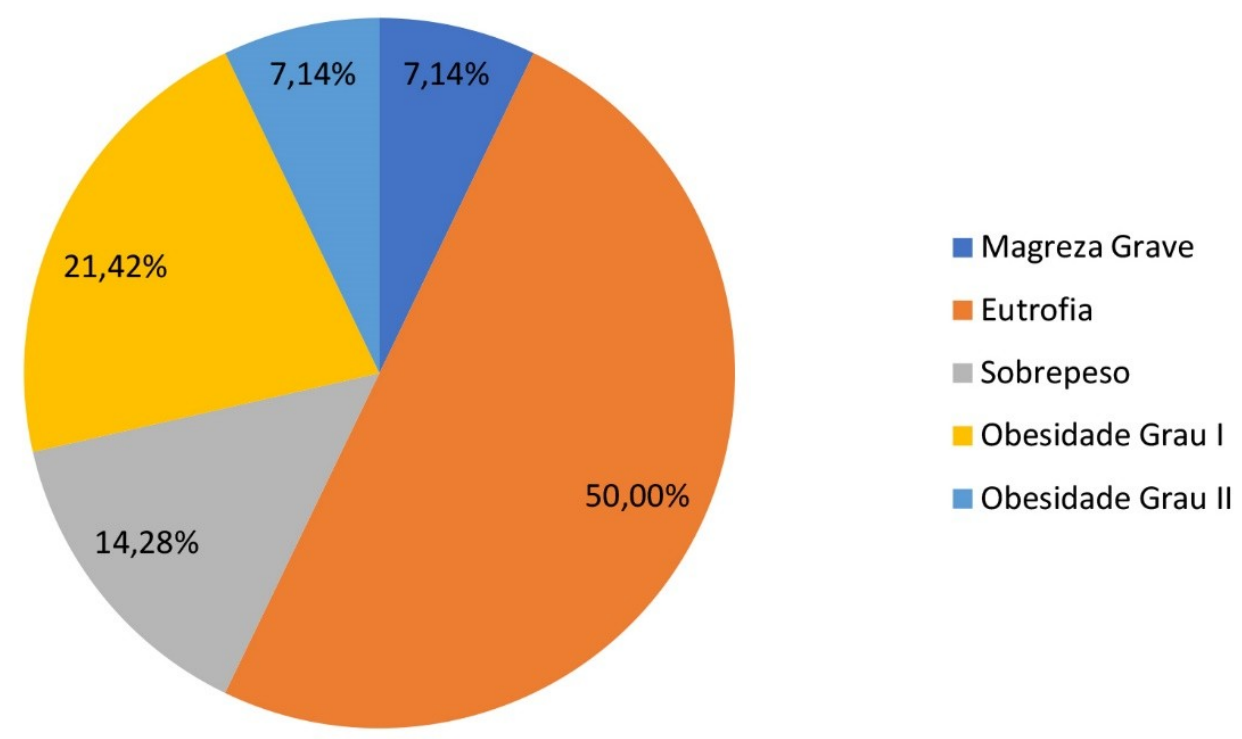

FIGURA 1 Estado Nutricional dos participantes da pesquisa na UFT, Palmas, 2018.

Fonte: Dados da pesquisa

Em consonância com os resultados deste estudo, Tonini et al. (2013) avaliaram o estado nutricional e hábitos alimentares de funcionários de uma instituição de ensino superior do oeste de Santa Catarina e obtiveram 39,3\% de excesso de peso. Mendes et al. (2018), em estudo recente com uma população de funcionários do restaurante universitário da Universidade Federal do Tocantins, revelaram percentuais consideráveis de sobrepeso (33\%) e obesidade (17\%).

Pequena porcentagem da amostra foi classificada com magreza de grau III $(7,14 \%, n=1)$, o que se atribuiu à presença de Doença Celíaca no paciente. Em relação ao risco cardiometabólico, avaliado pelo perímetro da cintura, obteve-se que $28,57 \%$ das mulheres apresentaram baixo risco, $7,14 \%$ apresentaram risco elevado e $14,28 \%$ risco muito elevado. Já entre os homens foram observados $28,57 \%$ dos indivíduos com baixo risco e $21,42 \%$ com risco muito elevado. Somam-se $35,70 \%$ de servidores em risco cardiometabólico muito elevado na amostra. A medida isolada da circunferência da cintura é suficiente para estabelecer o risco de comorbidades associadas ao sobrepeso, em diferentes graus, com risco substancialmente aumentado para dislipidemias, diabetes e doenças cardiovasculares (LOPES; REZENDE, 2019).

Resultados semelhantes foram encontrados em pesquisa com servidores do restaurante universitário da mesma instituição do presente estudo, onde foram observados $33 \%$ dos servidores com alto risco cardiometabólico (MENDES et al., 2018). Audi et al. (2016), avaliando servidores de uma instituição prisional, encontraram $49,6 \%$ dos indivíduos com risco cardiometabólico muito elevado.

No que se refere aos exames bioquímicos (tabela 1), no presente estudo $14,28 \%$ dos participantes apresentaram glicemia de jejum fora dos valores de referência. Em pesquisa sobre consumo alimentar de funcionários de uma unidade de alimentação e nutrição, obtiveram-se resultados semelhantes, com 12,1\% dos indivíduos acima da normalidade (OLIVEIRA et al., 2015). 
Apesar das alterações observadas na glicemia nesta pesquisa, os níveis glicêmicos não atingiram os critérios para diagnóstico de diabetes mellitus. Já em um estudo que avaliou a relação de indicadores antropométricos com a glicemia entre servidores universitários, o diabetes mellitus foi diagnosticado em 1,9\% dos indivíduos com elevação da glicemia em jejum (MACHADO; SICHIERI, 2002).

TABELA 1 Distribuição dos participantes conforme os níveis sanguíneos de colesterol total, triacilgliceróis e glicemia de jejum, UFT, Palmas, 2018.

\begin{tabular}{|c|c|}
\hline Dosagem Bioquímica & Percentuais \\
\hline \multicolumn{2}{|c|}{ Glicemia de Jejum } \\
\hline < 99 mg/dL (desejável) & $85,70 \%$ \\
\hline$\geq 99 \mathrm{mg} / \mathrm{dL}$ (elevada) & $14,28 \%$ \\
\hline \multicolumn{2}{|c|}{ Colesterol Total } \\
\hline Desejável (< 190 mg/dL) & $50,00 \%$ \\
\hline Alto ( $\geq 190 \mathrm{mg} / \mathrm{dL})$ & $50,00 \%$ \\
\hline \multicolumn{2}{|c|}{ Triacilgliceróis } \\
\hline Desejável (< 150 mg/dL) & $64,28 \%$ \\
\hline Limítrofe (150-199 mg/dL) & $21,42 \%$ \\
\hline Alto (200-499 mg/dL) & $7,14 \%$ \\
\hline Muito alto ( $\geq 500 \mathrm{mg} / \mathrm{dL}$ ) & $7,14 \%$ \\
\hline
\end{tabular}

Fonte: Dados da pesquisa

Barel et al. (2010), ao avaliarem a associação de risco para doenças cardiovasculares e qualidade de vida entre servidores da área de saúde, encontraram resultados semelhantes ao do presente estudo, estando prevalentes a hipercolesterolemia (49\%) e a hipertrigliceridemia (24\%). Outro estudo com servidores de uma universidade federal também evidenciou níveis elevados de colesterol total (49\%) e triacilglicerois (20\%) (OLIVEIRA et al., 2015).

Os parâmetros bioquímicos alterados sinalizam elevado risco cardiometabólico na amostra, pois o desequilíbrio da lipemia contribui para o desenvolvimento de aterosclerose (FALUDI et al., 2017). Os percentuais médios de ingestão diária de macronutrientes da amostra, baseados no recordatório de 24 horas, são mostrados na figura 2.

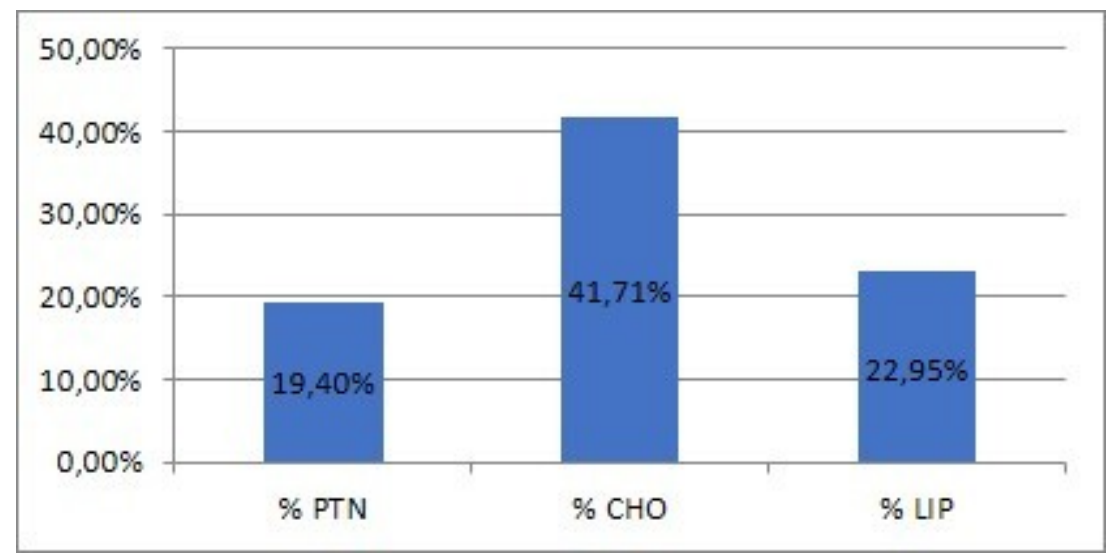

FIGURA 2 Percentuais médios de ingestão diária de macronutrientes pelos participantes da pesquisa na UFT, Palmas, 2018. 
A média de consumo energético diário foi de 1651,92 Kcal. Obteve-se também os consumos de proteína, carboidrato e lipídio que foram comparados às recomendações do intervalo aceitável de distribuição dos macronutrientes na dieta, proposto pelas Ingestões Dietéticas de Referência (IDR) (FERRIER, 2019).

A ingestão de proteína do grupo $(19,40 \%)$ esteve dentro da recomendação diária que é de $10 \%$ a $35 \%$ do valor energético total da dieta (VET). Para os carboidratos, a ingestão diária de $41,71 \%$ ficou abaixo da recomendação, que é $45 \%$ a $65 \%$ do VET. Em relação aos lipídios, o consumo médio do grupo $(22,95 \%)$ esteve dentro da recomendação, que é $20 \%$ a $35 \%$ do VET. Os valores médios de ingestão diária de micronutrientes pelos 14 servidores participantes da pesquisa estão descritos na tabela 2.

TABELA 2 Valores médios de ingestão diária de micronutrientes pelos 14 servidores participantes da pesquisa da UFT, Palmas 2018.

\begin{tabular}{|c|c|c|}
\hline Micronutrientes & Ingestão Média & Recomendações EAR \\
\hline Cálcio & $486,69 \mathrm{mg} / \mathrm{dia}$ & $1.142,86 \mathrm{mg} / \mathrm{dia}^{*}$ \\
\hline Colesterol & $458,60 \mathrm{mg} / \mathrm{dia}$ & $<200 \mathrm{mg} / \mathrm{dia}^{* *}$ \\
\hline Ferro & $13,64 \mathrm{mg} / \mathrm{dia}$ & $11,3 \mathrm{mg} / \mathrm{dia}^{*}$ \\
\hline Fibra & $20,44 \mathrm{~g} / \mathrm{dia}$ & $25 \mathrm{~g} / \mathrm{dia}^{* *}$ \\
\hline Fósforo & $1117,33 \mathrm{mg} / \mathrm{dia}$ & $837,5 \mathrm{mg} / \mathrm{dia}^{*}$ \\
\hline Magnésio & $243,29 \mathrm{mg} / \mathrm{dia}$ & $361,25 \mathrm{mg} / \mathrm{dia}^{*}$ \\
\hline Manganês & $2,68 \mathrm{mg} / \mathrm{dia}$ & $2 \mathrm{mg} / \mathrm{dia}^{*}$ \\
\hline Monoinsaturados & 8,80\% VET & $\leq 15 \% \mathrm{VET}^{* *}$ \\
\hline Poli-insaturados & 4,67\% VET & $\leq 10 \% \mathrm{VET}^{* *}$ \\
\hline Potássio & 2342,06 mg/dia & $4.600 \mathrm{mg} / \mathrm{dia}^{*}$ \\
\hline Saturada & $9,38 \%$ VET & $\leq 10 \% \mathrm{VET}^{* *}$ \\
\hline Selênio & $113,68 \mathrm{mg} / \mathrm{dia}$ & $53,12 \mathrm{mg} / \mathrm{dia}^{*}$ \\
\hline Sódio & $1309,28 \mathrm{mg} / \mathrm{dia}$ & $1437,5 \mathrm{mg} / \mathrm{dia}^{*}$ \\
\hline Gorduras Trans & $0,93 \mathrm{~g} / \mathrm{dia}$ & Não Consumir \\
\hline Retinol & $1207,67 \mathrm{mcg} / \mathrm{dia}$ & $787,5 \mathrm{mcg} / \mathrm{dia}^{*}$ \\
\hline Tiamina & $1,18 \mathrm{mg} / \mathrm{dia}$ & $1,11 \mathrm{mg} / \mathrm{dia}^{*}$ \\
\hline Cobalamina & $5,18 \mathrm{mcg} / \mathrm{dia}$ & $2,32 \mathrm{mcg} / \mathrm{dia}^{*}$ \\
\hline Riboflavina & $1,47 \mathrm{mg} / \mathrm{dia}$ & $1,16 \mathrm{mg} / \mathrm{dia}^{*}$ \\
\hline Niacina & $17,95 \mathrm{mg} / \mathrm{dia}$ & $14,75 \mathrm{mg} / \mathrm{dia}^{*}$ \\
\hline Piridoxina & $1,56 \mathrm{mg} / \mathrm{dia}$ & $1,35 \mathrm{mg} / \mathrm{dia}^{*}$ \\
\hline Ácido fólico & 174,46 mcg/dia & $387,5 \mathrm{mcg} / \mathrm{dia}^{*}$ \\
\hline Ácido ascórbico & 119,62 mg/dia & $77,5 \mathrm{mg} / \mathrm{dia}^{*}$ \\
\hline Calciferol & $1,00 \mathrm{mcg} / \mathrm{dia}$ & $6,8 \mathrm{mcg} / \mathrm{dia}^{*}$ \\
\hline Tocoferol & 10,68 mg/dia & $14,5 \mathrm{mg} / \mathrm{dia}^{*}$ \\
\hline Zinco & $14,88 \mathrm{mg} / \mathrm{dia}$ & $9,6 \mathrm{mg} / \mathrm{dia}^{*}$ \\
\hline
\end{tabular}

Fonte: Dados da Pesquisa

VET = valor energético total da dieta. *Média de recomendações segundo a Dietary Reference Intakes - EAR (DRI para grupos), considerando homens e mulheres adultos na faixa etária de 19 a 50 anos. ${ }^{* *}$ Recomendações segundo a IV Diretriz Brasileira sobre Dislipidemias e Prevenção da Aterosclerose. 
Foi identificado que o consumo de fibras não atingiu a recomendação diária (FERRIER, 2019), sendo que o consumo de fibras contribui para a redução do colesterol, promove efeito protetor contra formação de ateromas e, consequentemente, colabora para a prevenção de doenças cardiovasculares e câncer (FALUDI et al., 2015).

Uma alimentação saudável, rica em fibras, implica no consumo de frutas e verduras, porém vem se observando que a população tem reduzido o consumo desses alimentos e aumentado o consumo de produtos industrializados. A pesquisa "Vigilância de Fatores de Risco e Proteção para Doenças Crônicas por Inquérito Telefônico" (VIGITEL), realizada no ano de 2016, abrangeu 27 capitais e Distrito Federal, e constatou que o consumo de frutas e hortaliças em cinco ou mais dias da semana é observado em apenas 35,2\% dos indivíduos (BRASIL, 2017).

O consumo de sódio do grupo esteve abaixo da recomendação, corroborando com a informação fornecida quanto ao hábito de adicionar sal na refeição pronta, em que $85,3 \%$ dos participantes relataram não terem esse hábito. A redução da ingestão de sal de todas as formas é desejável, como forma de prevenção primária e de tratamento não medicamentoso da hipertensão arterial e da síndrome metabólica (CEMBRANEL et al., 2016).

A ingestão das vitaminas calciferol, tocoferol e ácido fólico, dos minerais potássio, magnésio e cálcio ficaram inferiores às recomendações. Já níveis superiores aos recomendados foram observados para as vitaminas retinol, tiamina, niacina, ácido ascórbico e para os minerais ferro, selênio, fósforo, manganês e zinco, além do colesterol. O magnésio é um micronutriente importante e protetor contra doenças cardiovasculares, contribuindo para redução da rigidez arterial. Estudos tem explorado também efeitos hipotensores deste mineral (SCHUTTEN et al., 2018).

Quanto às vitaminas, a baixa ingestão de tocoferol foi observada no presente estudo, bem como verificada na população de servidores do restaurante universitário da UFT e em população residente no Sul do País (TONINI et al., 2013; MENDES et al., 2018). Nesse sentido a escassez de tocoferol na alimentação eleva o risco cardiovascular, conforme estudos que demonstraram efeito cardioprotetor dessa vitamina (HU et al., 2015). Da mesma forma, as baixas ingestões de calciferol e de cálcio têm sido associadas ao aumento da prevalência de doenças cardiovasculares e osteoporose (CARVALHO et al., 2015). Além disso, os níveis séricos de colecalciferol têm sido inversamente associados ao índice de massa corporal e à pressão arterial na população em geral (MIDTBO et al., 2020).

A ingestão alimentar de colesterol superou os níveis recomendados por dia, o que explica o perfil de hipercolesterolemia dos servidores e predispõe ao grande risco de desenvolvimento de doenças cardiovasculares (FERRIER, 2019).

\section{CONCLUSÃO}

O estudo retratou uma realidade desfavorável, pelos parâmetros antropométricos e dietéticos, aos servidores participantes da pesquisa, que afirmados pelos exames bioquímicos, revelou um público predisposto a doenças cardiovasculares e doenças crônicas não transmissíveis.

Elevados índices de excesso de peso e de circunferência da cintura, associados a hipercolesterolemia e hipertrigliceridemia, mostraram que há necessidade de se praticar hábitos mais saudáveis nessa população, inserindo exercícios físicos ao cotidiano e melhorando os hábitos alimentares, pela redução da ingestão de gorduras e aumento do consumo de frutas e verduras, afim de aumentar a ingestão de fibras alimentares, vitaminas e mineiras. 


\section{REFERÊNCIAS}

ARAÚJO, T. P.; REZENDE, F. A. C. Obesidade e Síndrome Metabólica. In: LOPES, E. C.; PEREIRA, R. J.; REZENDE, F. A.C. Nutrição do Adulto: diretrizes para a assistência ambulatorial. 1.ed. Palmas: Editora da Universidade Federal do Tocantins, 2019.172p.

AUDI, C. A.; SANTIAGO, S. M.; ANDRADE, M. G. G.; FRANCISCO, P. M. S. B. Fatores de risco para doenças cardiovasculares em servidores de instituição prisional: estudo transversal. Epidemiologia e Serviços de Saúde, v. 25, n. 32, p. 301-310, 2016.

BAREL, M.; LOUZADA, J. C. A.; MONTEIRO, H. L.; AMARAL, S. L. Associação dos fatores de risco para doenças cardiovasculares e qualidade de vida entre servidores da saúde. Revista Brasileira de Educação Física e Esporte, v. 24, n. 2, p. 293-303, 2010. Disponível em: < http://www.scielo.br/scielo.php?script=sci_arttext\&pid=S180755092010000200012>. doi: 10.1590/S1807-55092010000200012

BRASIL. Instituto Brasileiro de Geografia e Estatística (IBGE). Pesquisa de Orçamentos Familiares 2008-2009: análise do consumo alimentar no Brasil. Rio de Janeiro: IBGE; 2011a. 150 p. Disponível em: < https://biblioteca.ibge.gov.br/visualizacao/livros/ liv50063.pdf>.

BRASIL. Instituto Brasileiro de Geografia e Estatística. Pesquisa de Orçamentos Familiares 2008-2009: tabelas de composição nutricional dos alimentos consumidos no Brasil. Rio de Janeiro: Instituto Brasileiro de Geografia e Estatística; 2011b. Disponível em: < https://biblioteca.ibge.gov.br/visualizacao/livros/liv50002.pdf>.

BRASIL. Secretaria de Vigilância em Saúde. Secretaria de Gestão Estratégica e Participativa. Vigitel Brasil 2016: vigilância de fatores de risco e proteção para doenças crônicas por inquérito telefônico. Brasília (DF): Ministério da Saúde; 2017. 157 p. Disponível em: < https://portalarquivos2.saude.gov.br/images/pdf/2018/marco/02/vigitel-brasil-2016.pdf>.

BRAVIM, A. F.; ROCHA, A. M. S.; FERREIRA, A. M. R. S.; GOMES, A. C.; DANTAS, A. et al. Plano de Ações Estratégicas para enfretamento das Doenças Crônicas e Agravos Não Transmissíveis no Espírito Santo. 1. ed. Gráfica Aquarius: Vitória, 2016. Disponível em: <https://saude.es.gov.br/Media/sesa/DANTS/Plano\%20DANT \%C2\%B4s\%20p\%20p\%C3\%A1gina\%2020\%2012\%202017.pdf>.

CARVALHO, L. S.; SPOSITO, A. C. Vitamin D for the prevention of cardiovascular disease: are we ready for that? Atherosclerosis, v. 241, n. 2, p. 729-40, 2015. Disponível em: < https://www.ncbi.nlm.nih.gov/pubmed/26135478>. doi: 0.1016/ j.atherosclerosis.2015.06.034.

CEMBRANEL, F.; GONZÁLEZ-CHICA, D. A.; D'ORSI, E. Inadequações na ingestão dietética de micronutrientes por homens e mulheres residentes no Sul do Brasil: Estudo EpiFloripa Adultos 2012. Cadernos de Saúde Pública, v. 32, n. 8, e00164015, 2016. Disponível em: < http://www.scielo.br/scielo.php?pid=S0102311X2016000806001\&script=sci_abstract\&tIng=pt>. doi: 10.1590/0102-311X00164015. 
CHAVES, G.; BRITEZ, N.; MUNZINGER, J.; UHLMANN, L.; GONZALEZ, G. et al. Educação para um Estilo de Vida Saudável Melhora Sintomas e Fatores de Risco Cardiovasculares - Estudo AsuRiesgo. Arquivos Brasileiros de Cardiologia, v. 104, n. 5, p. 347-55, 2015. Disponível em: < http://www.scielo.br/scielo.php?pid=S0066782X2015005050021\&script=sci_arttext\&tlng=pt>. doi: 10.5935/abc.20150021

FALUDI, A. A.; IZAR, M. C. O.; SARAIVA, J. F. K.; CHACRA, A. P. M.; BIANCO, H. T. et al. Atualização da Diretriz Brasileira de Dislipidemias e Prevenção da Aterosclerose 2017. Arquivos Brasileiros de Cardiologia, v. 109, n. 2, supl. 1, p. 1-76, 2017. Disponível em: < http://publicacoes.cardiol.br/2014/diretrizes/2017/02_DIRETRIZ_DE_DISLIPIDEMIAS.pdf>. doi: 10.5935/abc.20170121.

FERREIRA, J. S.; DIETTRICH, S. H. C.; PEDRO, D. A. Influência da prática de atividade física sobre a qualidade de vida de usuários do SUS. Saúde em Debate, v. 39, n. 106, p. 792-801, 2015. Disponível em: < http://www.scielo.br/scielo.php?pid=S010311042015000300792\&script=sci_abstract\&tlng=pt>. doi: 10.1590/01031104201510600030019

FERRIER, D. Bioquímica Ilustrada. 7.ed. São Paulo: Artmed, 2019. 530p.

HU, X. X.; FU, L.; LI, Y.; LIN, Z. B. LIU, X.; WANG, J. F. et al. The cardioprotective effect of Vitamin $E$ (AlphaTocopherol) is strongly related to age and gender in mice. PLoS One, v.10, n. 9, e0137405, 2015. Disponível em: < https://www.ncbi.nlm.nih.gov/pubmed/26331272>. doi: 10.1371/journal.pone.0137405.

LELIS, C. T.; TEIXEIRA, K. M. D.; SILVA, N. M. A inserção feminina no mercado de trabalho e suas implicações para os hábitos alimentares da mulher e de sua família. Saúde em Debate, v. 36, n. 95, p. 523-32, 2012. Disponível em: < http://www.scielo.br/scielo.php?pid=S0103-11042012000400004\&script=sci_abstract\&tlng=pt>. doi: 10.1590/ S0103-11042012000400004

LOPES, E.C.; REZENDE, F. A. C. Avaliação antropométrica de adultos. In: LOPES, E. C.; PEREIRA, R. J.; REZENDE, F. A.C. Nutrição do Adulto: diretrizes para a assistência ambulatorial. 1.ed. Palmas: Editora da Universidade Federal do Tocantins, 2019.172p.

MACHADO, P. A. N.; SICHIERI, R. Relação cintura-quadril e fatores de dieta em adultos. Revista de Saúde Pública, v. 36, n. 2, p. 198-204, 2002. Disponível em: < http:// www.scielo.br/scielo.php?pid=S0034-

89102002000200012\&script=sci_abstract\&tlng=pt $>. \quad$ doi: $\quad$ 10.1590/S003489102002000200012.

MALTA, D. C.; ANDRADE, S. S. C. A.; OLIVEIRA, T. P.; MOURA, L.; PRADO, R. R. et al. Probabilidade de morte prematura por doenças crônicas não transmissíveis, Brasil e regiões, projeções para 2025. Revista Brasileira de Epidemiologia, v. 22, E190030, p. $1-13,2019$. Disponível em:< http://www.scielo.br/scielo.php? script=sci_abstract\&pid=S1415-790X2019000100428\&lng=en\&nrm=iso\&tlng=pt>. doi: $10.1590 / 1980-549720190030$ 
MENDES, A. K. F.; CARVALHO, A. K. C.; JUSTINO, M. G.; GRATÃO, L. H. A.; PEREI$\mathrm{RA}, \mathrm{R}$. J. Triagem nutricional e riscos cardiometabólicos nos funcionários de um restaurante universitário. Demetra - Alimentação, Nutrição \& Saúde, v. 13, n. 1, p. 485502, 2018. Disponível em:< https://www.e-publicacoes.uerj.br/index.php/demetra/article/view/31559/25648>. doi: 10.12957/demetra.2018.31559

MENEGASSI, B. Mastigação: reflexões e interfaces com a saúde. Ensaios e Diálogos, v.11, p. 26-29, 2017. Disponível em: < https://www.abrasco.org.br/site/wp-content/uploads/2017/11/ENSAIOS-DI\%C3\%81LOGOS-5_ARTIGO-7.pdf>.

MIDTBO, L. K.; NYGAARD, L. B.; MARKHUS, M. W.; KJELLEVOLD, M.; LIE, O. et al. Vitamin $D$ status in preschool children and its relations to vitamin $D$ sources and body mass index-Fish Intervention Studies-KIDS (FINS-KIDS). Nutrition, v. 70, 110595, 2020. Disponível em: < https://www.sciencedirect.com/science/article/abs/pii/ S0899900719301790>. doi: 10.1016/j.nut.2019.110595

NEUENSCHWANDER, M.; BALLON, A.; WEBER, K. S.; NORAT, T.; AUNE, D. et al. Role of diet in type 2 diabetes incidence: umbrella review of meta-analyses of prospective observational studies. Journal of the British Medical Association, v. 365, I2368, 2019. Disponível em: < https://www.bmj.com/content/366/bmj.I2368>. doi:10.1136/ bmj. 12368

NEPA - Núcleo de Estudos e Pesquisas em Alimentação. Universidade Estadual de Campinas. Tabela Brasileira de Composição de Alimentos [TACO]. 4.ed. Campinas: NEPA /UNICAMP, 2011. 161 p. Disponível em: < http://www.nepa.unicamp.br/taco/tabela.php?ativo=tabela>.

OLIVEIRA, J. E. P.; MONTENEGRO JUNIOR, R. M.; VENCIO, S. Diretrizes da Sociedade Brasileira de Diabetes 2017-2018. São Paulo: Clannad; 2017.

OLIVEIRA, R. A. R.; AMORIM, P. R. S.; MOREIRA, O. C.; AMORIM, W.; COSTA, E, G. et al. Relação de indicadores antropométricos com glicemia entre servidores universitários. Revista de Ciências Médicas, v. 24, n.1, p. 19-28, 2015. Disponível em: <https:// seer.sis.puc-campinas.edu.br/seer/index.php/cienciasmedicas/article/view/2357>. doi: 10.24220/2318-0897v24n1a2357.

PHILIPI, S. T. Tabela de Composição de Alimentos: Suporte para Decisão Nutricional. 4 ed. São Paulo: Metha; 2015. 164 p.

RAMIRES, E. K. N. M.; MENEZES, R. C. E.; LONGO-SILVA, G.; SANTOS, T. G.; MARINHO, P. M. et al. Prevalência e Fatores Associados com a Síndrome Metabólica na População Adulta Brasileira: Pesquisa Nacional de Saúde - 2013. Arquivos Brasileiros de Cardiologia, v. 110, n. 5, p. 455-66, 2018. Disponível em: <http://www.scielo.br/ scielo.php?script=sci_arttext\&pid=S0066-

782X2018000500455\&lng=en\&nrm=iso\&tlng=pt>. doi: 10.5935/abc. 20180072

SCHUTTEN, J. C.; JOOSTEN, M.M.; BORST, M. H.; BAKKER, S. J. L. Magnesium and Blood Pressure: A Physiology-Based Approach. Advances in Chronic Kidney Disease, v. 25, n. 3, p. 244-50, 2018. Disponível em: < https://www.ncbi.nlm.nih.gov/ pubmed/29793663>. doi: 10.1053/j.ackd.2017.12.003 
TONINI, E.; BROLL, A. M.; CORREAA, E. N. Avaliação do estado nutricional e hábito alimentar de funcionários de uma instituição de ensino superior do oeste de Santa Catarina. O Mundo da Saúde, v. 37, n. 3, p. 268-279, 2013. Disponível em: < http://bvsms.saude.gov.br/bvs/artigos/mundo_saude/avaliacao_estado_nutricional_habito_alimentar.pdf>.

UNITED STATES DEPARTMENT OF AGRICULTURE. Nutrient database for standard reference. 2012. Disponível em: < https://ndb.nal.usda.gov/>.

VUORI, I. World Health Organization and Physical Activity. Progress in Preventive Medicine, v. 3, e0012, p. 1-7, 2018. Disponível em: < https://journals.Iww.com/progprevmed/FullText/2018/01000/

World_Health_Organization_and_Physical_Activity.1.aspx>. doi: 10.1097/ pp9.0000000000000012 